Abstract

\title{
Using the Continuous Shape and Symmetry Measures to Correlate Atomic Structure and Chemical Properties ${ }^{+}$
}

\author{
Miquel Llunell 1,2 \\ 1 Department of Materials Science and Physical Chemistry, University of Barcelona, 08007 Barcelona, Spain; \\ llunell@ub.edu \\ 2 Institute of Theoretical and Computational Chemistry, University of Barcelona (IQTCUB), \\ 08007 Barcelona, Spain \\ + Presented at Symmetry 2017-The First International Conference on Symmetry, Barcelona, Spain, \\ 16-18 October 2017.
}

Published: 1 February 2018

In the field of structural chemistry, many geometrical and structural measures of molecules, clusters or crystals have been largely used to correlate them with physical or chemical properties. The main advantage of these correlations is the fact that no information about the electronic structure should be known, since just topological measures are considered. Considering shape or symmetry as a continuous real value property of a given topological structure instead of a binary property, many different shape and symmetry measures have been proposed. In this talk, the Continuous Shape Measures (CShM) and the Continuous Symmetry Measures (CSM) and some of their applications in structural chemistry will be introduced. Despite its apparent simplicity, the structural analysis of the atomic positions by means of these shape or symmetry measures has demonstrated to be a useful tool for the structure-property correlation analysis.

(C) 2018 by the authors. Licensee MDPI, Basel, Switzerland. This article is an open access article distributed under the terms and conditions of the Creative Commons Attribution (CC BY) license (http://creativecommons.org/licenses/by/4.0/). 